\title{
Effect of Radiation on the Performance of the Stomatognathic System - a Pilot Study
}

\author{
Andre MM. Dante ${ }^{1}$, Carla MD. Santos ${ }^{1}$, Marcelo Palinkas ${ }^{1 *}$,Joao BM. Lellis ${ }^{1}$, Selma Siessere ${ }^{1}$, \\ Marisa Semprini $^{1}$, Helio M. Tanimoto ${ }^{2}$, Bruno Ferreira ${ }^{1,3}$, Gabriel PD. Silva ${ }^{1,3}$, Regiane AF. Lellis ${ }^{1}$ \\ and Simone $\mathrm{CH}$. Regalo ${ }^{1}$ \\ ${ }^{1}$ Department of Morphology, Stomatology and Basic Pathology, Ribeirao Preto Dental School, University of Sao Paulo, Ribeirao Preto, Brazil \\ ${ }^{2}$ Department of Dentistry, Barretos Cancer Hospital - Pio XII, Barretos, Brazil \\ ${ }^{3}$ Department of Physiotherapy, University Center UNIFAFIBE, Bebedouro; Brazil
}

Received: July 31, 2014; Accepted: September 15, 2014; Published: October 03, 2014

*Corresponding author: Marcelo Palinkas, Department of Morphology, Stomatology and Basic Pathology, Ribeirao Preto Dental School, University of Sao Paulo, Ribeirao Preto, Brazil, Tel: +55-163-602-0182; E-mail: marcelopalinkas@hotmail.com

\begin{abstract}
The purpose of this study was to evaluate the Electromyography (EMG), thickness of the masseter and temporal muscles, maximal bite force in individuals with head and neck cancer treated with radiotherapy. The patterns of the muscle activity were evaluated by means of the EMG recordings of the masseter and temporal muscles; bilaterally during the following clinical conditions: rest, protrusion, right and left lateral excursions and Maximum Habitual Intercuspation (MHI). Muscle thickness was evaluated using ultrasound at rest and in MHI. Maximal bite force in the right and left molar regions of 10 fully dentate individuals with head and neck cancer was evaluated after radiotherapy and compared with a control group. The EMG means showed that at rest, protrusion, and left lateral excursion, there were statistically significant differences $(p<0.05)$. On evaluation with ultrasound, the muscle thickness values were higher for individuals with head and neck cancer at rest and in MIH, with statistically significant results found only for the right temporal during the MIH. Participants with head and neck cancer treatment were evaluated in the present study showed higher electromyographic activity when compared with the control group for all clinical situations. The treatment used with patients undergoing head and neck cancer increased the electromyographic activity, the muscle thickness, and the bite force when compared to control subjects. The results suggest a muscle dysfunction of the stomatognathic system in individuals with head and neck cancer treatment evaluated in the present study.
\end{abstract}

Keywords: Head and neck cancer; Electromyography; Ultrasound; Bite Force; Stomatognathic System

\section{Introduction}

Cancer is considered as an extremely complex genetic disease, which results from concomitant changes in genes usually related to proliferation, differentiation and cell death. Head and neck tumors include a wide variety of malignancies that originates in the mucosa of the upper aero digestive tract, and account for approximately $5 \%$ of all new diagnosed tumors [1,2].

Dentistry plays an important role in the various therapeutic phases against cancer, either before surgery, where a prior assessment can effectively reduce complications from infectious or chronic inflammatory processes of oral origin, or in prevention of oral sequelae such as Osteoradionecrosis (ORN), which occurs as a complication of radiation therapy [3].

Radiotherapy to the head and neck can cause temporary side effects and several adverse reactions in soft and hard tissues of the oral cavity and in the adjacent areas. Sometimes it can cause immediate and/or long-lasting side effects that develop months or even years later which are associated with the dose applied, the type of radiation, and the characteristics of the tissue cells involved. When a patient undergoes surgery combined with radiotherapy, functional and aesthetic rehabilitation may be necessary [4-6].

The vulnerability of the stomatognathic functions due to the lesions caused by head and neck cancer can impair mastication, swallowing, speech, facial appearance and salivary flow. Therefore, the types and intensity of these side effects may vary from person to person, even when it is the same lesion and the same stage of disease [7].

The knowledge of the organization and morpho-functional efficiency of the stomatognathic system in individuals with head and neck cancer, who have undergone radiation treatment, is extremely important to understand the functions of the masticatory muscles. This fact has stimulated the development of this research, which aims to collaborate with professionals in the area and also with individuals suffering from this disease.

\section{Material and Methods}

\section{Patients}

A hundred and twenty seven individuals undergoing post radiotherapy were evaluated at the Hospital de Cancer de Barretos/Pio XII (Sao Paulo-Brazil), during the period of 2011 and 2012 and twenty subjects, between the ages of 15 to 55 years $(+3.0)$ were included in the final sample according to the inclusion and exclusion criteria for participation. The sample 
was selected by means of anamneses, clinical exams, and possible Temporomandibular Dysfunction symptoms (TMD). All subjects need to be fully dentate, Caucasian, nasal breathers, had normal occlusion and no parafunctional habits and symptoms of Temporomandibular Dysfunction (TMD).

The patients were divided into two groups: Group I ( $n=$ 10), patients with head and neck cancer in different regions (retromolar trigone, tongue, salivary gland, larynx, skin of the face, skull, brain and lymph nodes on the left side), stages III and IV, classified as resectable or not, treated with six month of radiotherapy, and Group II $(n=10)$, healthy individuals (Control) consisted of patients selected by comparing subject to subject (age, gender and Body Mass Index - BMI).

This study was approved by the Research Ethics Committee of the Hospital de Câncer de Barretos/São Paulo/Brazil (process number 369/2010) in compliance with the Resolution 466/12 of the Brazilian National Health Council. The patients and those responsible for minor participants were informed about the experiment and agreed to take part in the study by signing an informed consent form.

\section{Electromyographic analysis}

For the EMG recording, the Myosystem-BR1 apparatus DataHomins Tecnologia Ltd was used with simultaneous acquisition, common grounding to all channels, low-pass filters of $10 \mathrm{~Hz}-\mathrm{kHz}$, channel input impedance of $10 \mathrm{G} \Omega$ in differential mode, 12 bytes of dynamic resolution range, an amplitude band of $-10 \mathrm{~V}$ to $+10 \mathrm{~V}$ and a channel and a channel sampling frequency of $2 \mathrm{kHz}$. For signal visualization and processing, the Myosystem I version 3.5 software was used, which allowed the analogue signals to be amplified with a $1000 \mathrm{X}$ gain, filtered by $0.01-1.5$ $\mathrm{kHz}$ bandpass filter and sampled by 12-b A/D converter with an acquisition frequency of $2 \mathrm{kHz}$. Surface differential active electrodes (two 1-mm long and 2-mm wide silver-chloride bars, separated by a distance of $10 \mathrm{~mm}$, with input impedance of 10 $\mathrm{G} \Omega$ and a common mode rejection ratio of $130 \mathrm{db}$ at $60 \mathrm{~Hz}$ ) were used in this study.

The skin area where the electrodes were placed was cleaned with alcohol to reduce skin impedance, before positioning the electrodes on the masticatory muscles according to the recommendations of SENIAN, 1999 [8]. A stainless steel circular electrode was fixed to the skin in the wrist region and used as a reference electrode.

The evaluation of the muscle activity was performed using electromyographic recordings of masseter and temporal muscles, bilaterally, in the following mandibular postural conditions: rest (4 s), dental clenching in voluntary contraction (4 s) and dental clenching with parafilm $\mathrm{M}^{\circledR}$ (Pechinery Plastic Packaging, Batavia, IL, USA) (4 s); in the following clinical conditions: maximal right lateral excursion (10 s), maximal left excursion (10 s) with dental contact, and maximal mandibular protrusion with dental contact (10 s).

During EMG recording, the patient sat on a comfortable chair, with head and trunk erect, the soles of the feet on the ground and the hands resting on the thighs. The participants were given detailed instructions prior to the examination, and were asked to be relaxed, breathing slowly.

\section{Ultrasound analysis}

Muscle thickness analyses were performed with a portable SonoSite Titan ultrasound tool, nationalized, using a $56 \mathrm{~mm} / 10$ $\mathrm{MHz}$ linear-array transducer. During the exam, an optimized imaging was obtained. Initially, the temporal and masseter muscles were located by palpation and transducer movement. The participants remained seated, leaning on the backrest with the head unrestrained.

Three exams were performed in the following clinical condition: rest and maximal voluntary contraction, with an interval of 2 min between each acquisition for the participants to relax and rest. For the exam, the linear transducer was placed in the middle of the muscle, transversally to the muscle fibers. Ultrasound images were obtained from temporal and masseter muscles (right and left). Measures were taken directly over the image in the moment of its acquisition, with an approximation of $0.1 \mathrm{~mm}$.

\section{Bite force}

The bite force recordings were taken with a digital mouthadapted $1000 \mathrm{~N}$ dynamometer; model IDDK (Kratos, Cotia, SP, Brazil). The device has two arms with Teflon disks on each end, over which the bite force is applied and recorded.

The measures were taken from the mandibular right and left first molar regions, where the maximal bite force occurs $[9,10]$. During the procedure, the patients sat on a chair, with the arms extended along the body and the hands lying on the thighs, and were instructed and trained on how to bite the dynamometer, to ensure the reliability of the procedure. For each patient, the apparatus was cleaned with alcohol and disposable latex finger cots (Wariper-SP, Brazil) were positioned on the biting arms as a biosecurity measure.

Three measures were taken from each side, with a 2-min rest interval between records. The highest out of three records was regarded as the maximal bite force [11-13].

\section{Statistical analysis}

The data were submitted to statistical analysis using the SPSS software, version 19.0 for Windows (SPSS Inc.; Chicago, IL, USA). A descriptive analysis was performed for each variable (means, standard deviation). A level of significance of 95\% ( $\mathrm{p}<$ $0.05)$ was set. The EMG signals in mandibular postural conditions were normalized during the clinical condition of dental clenching with parafilm $\mathrm{M}^{\circledR}$ and processed using Myosystem - Br1 version 3.56 software. After digitization, the signals were analogically amplified (with $1000 \mathrm{X}$ gain), filtered $(0.01$ to $1.5 \mathrm{kHz}$ bandpass filter), and sampled by a 12-b A/D converter with an acquisition frequency of $2 \mathrm{kHz}$.

The data obtained from the electromyographic analyses, ultrasound analysis and maximal molar bite force were compared by means of independent $t$-test between the groups. 


\section{Results}

The electromyographic data normalized for the muscles analyzed in each clinical condition for the two groups are shown in Table 1.

At rest, Group 1 presented higher EMG activity of the Right Masseter (RM), Left Masseter (LM) and Right Temporal (RT) muscles when compared to Group II, and there was statistically significance for the RM and LT muscles $(p<0.05)$. The temporal muscles showed hyperactivity when compared to both masseter muscles.

For protrusion, Group I showed higher EMG activity of the bilateral masseter and temporal muscles, when compared to Group II, and there was statistically significant difference for the RM, LM and RT ( $p<0.05)$. The masseter muscles presented hyperactivity when compared to both temporal muscles.

For lateral excursions, there was higher activation of the RT and LM muscles in right lateral excursion, and the EMG acitvity was higher in LT and RM in left lateral excursion during the analysis of both groups. Group I presented higher EMG activity in the masticatory muscles in right lateral excursion, when compared to values obtained from Group II. However, there were no statistically significant differences $(p<0.05)$.

On the data obtained during left lateral excursion, it was possible to observe greater EMG activity of masseter muscles and left temporal muscle in Group I. However, when compared; data were found to be statistically significant just for the RM muscle $(p<0.05)$.

During maximal dental clenching, the temporal muscles showed hyperactivity when compared to the masseter muscles (RM and LM) in both groups. There were no statistically significant differences in any of the comparisons made $(p<0.05)$.

The thickness mean values of the masseter and temporal muscles at rest and at maximal voluntary contraction for Groups I and II are presented in Table 2 . The results obtained from the ultrasound exam in the rest condition showed higher values in the thickness of these muscles in Group I when compared to Group II. However, the results showed no statistical significance in any of the comparisons made $(p<0.05)$.

The highest bite force values of the right and left molar are presented in Table 3. The independent t-test showed no statistically significant differences between Groups I and II.

\section{Discussion}

According to the National Cancer Institute of the Brazilian/ Ministry of Health, the sites most affected by head and neck cancer are the oral cavity, esophagus, larynx, skin and lymph vessels. In 2012, the estimated number of new cases of cancer in the oral cavity was 9,990 in men and 4,180 in women; and for larynx cancer, it was 6,110 individuals (men and women). In 2010 , the number of deaths totaled 3,189 men and 429 women [14]. The estimated number of cases suggests that the number of individuals with cancer is increasing as well as the search for information, diagnosis and treatment.
Table 1: Normalized electromyiographic means (microvolts/seconds) between Group I and Group II (t-test): RMS values for the Right Masseter (RM), Left Masseter (LM), Right Temporal (RT), Left Temporal (LT) muscles under different clinical conditions.

\begin{tabular}{|c|c|c|c|c|}
\hline \multirow[b]{2}{*}{ Clinical Condition } & \multirow[b]{2}{*}{ Muscles } & \multicolumn{2}{|c|}{ Groups } & \multirow[b]{2}{*}{$p$} \\
\hline & & I & II & \\
\hline \multirow{4}{*}{ Rest } & $\mathrm{RM}$ & $0.07 \pm 0.01$ & $0.03 \pm 0.00$ & 0.02 \\
\hline & LM & $0.07 \pm 0.02$ & $0.06 \pm 0.15$ & 0.65 \\
\hline & $\mathrm{RT}$ & $0.09 \pm 0.02$ & $0.08 \pm 0.15$ & 0.46 \\
\hline & LT & $0.08 \pm 0.19$ & $0.09 \pm 0.26$ & 0.00 \\
\hline \multirow{4}{*}{ Protrusion } & $\mathrm{RM}$ & $0.28 \pm 0.06$ & $0.10 \pm 0.02$ & 0.00 \\
\hline & LM & $0.32 \pm 0.07$ & $0.15 \pm 0.04$ & 0.01 \\
\hline & RT & $0.19 \pm 0.07$ & $0.07 \pm 0.16$ & 0.02 \\
\hline & LT & $0.15 \pm 0.05$ & $0.10 \pm 0.02$ & 0.32 \\
\hline \multirow{4}{*}{ Right laterality } & $\mathrm{RM}$ & $0.29 \pm 0.04$ & $0.10 \pm 0.01$ & 0.12 \\
\hline & LM & $0.33 \pm 0.06$ & $0.21 \pm 0.02$ & 0.16 \\
\hline & RT & $0.25 \pm 0.05$ & $0.21 \pm 0.04$ & 0.86 \\
\hline & LT & $0.14 \pm 0.03$ & $0.15 \pm 0.04$ & 0.52 \\
\hline \multirow{4}{*}{ Left laterality } & $\mathrm{RM}$ & $0.22 \pm 0.07$ & $0.06 \pm 0.00$ & 0.01 \\
\hline & LM & $0.17 \pm 0.06$ & $0.06 \pm 0.01$ & 0.11 \\
\hline & RT & $0.07 \pm 0.01$ & $0.08 \pm 0.01$ & 0.77 \\
\hline & LT & $0.15 \pm 0.05$ & $0.14 \pm 0.04$ & 0.75 \\
\hline \multirow{4}{*}{$\begin{array}{c}\text { Maximal Voluntary } \\
\text { Contraction }\end{array}$} & $\mathrm{RM}$ & $0.77 \pm 0.06$ & $0.81 \pm 0.10$ & 0.08 \\
\hline & LM & $0.81 \pm 0.08$ & $0.79 \pm 0.04$ & 0.21 \\
\hline & RT & $0.91 \pm 0.10$ & $0.86 \pm 0.07$ & 0.78 \\
\hline & LT & $0.87 \pm 0.09$ & $0.90 \pm 0.07$ & 0.90 \\
\hline
\end{tabular}

Significant $(p<0.05)$

Table 2: Comparison of muscle thickness measured by ultrasound between Group I and Group II ( $t$-test): data in $\mathrm{cm}$ for the Right Masseter (RM), Left Masseter (LM), Right Temporal (RT) and Left Temporal (LT) muscles under the different the clinical conditions.

\begin{tabular}{|c|c|c|c|c|}
\hline & & \multicolumn{2}{|c|}{ Groups } & \\
\hline \multirow{3}{*}{ Clinical Condition } & Muscles & I & II & $p$ \\
\hline \multirow{3}{*}{ Rest } & RM & $1.09 \pm 0.07$ & $0.95 \pm 0.03$ & \multirow{2}{*}{0.12} \\
\cline { 2 - 5 } & LM & $1.09 \pm 0.04$ & $0.96 \pm 0.04$ & \\
\cline { 2 - 5 } & RT & $0.82 \pm 0.05$ & $0.78 \pm 0.04$ & \multirow{2}{*}{0.60} \\
\cline { 2 - 5 } & LT & $0.85 \pm 0.05$ & $0.79 \pm 0.03$ & \\
\hline \multirow{3}{*}{$\begin{array}{c}\text { Maximal Voluntary } \\
\text { Contraction }\end{array}$} & RM & $1.28 \pm 0.07$ & $1.18 \pm 0.03$ & \multirow{2}{*}{0.31} \\
\cline { 2 - 5 } & LM & $1.37 \pm 0.06$ & $1.16 \pm 0.06$ & \\
\cline { 2 - 5 } & RT & $0.95 \pm 0.07$ & $0.82 \pm 0.04$ & \multirow{2}{*}{0.43} \\
\cline { 2 - 5 } & LT & $0.94 \pm 0.06$ & $0.86 \pm 0.02$ & \\
\hline
\end{tabular}

Significant $(p<0.05)$

It was difficult to select individuals with head and neck cancer that met our inclusion criteria i.e. patients with complete dentition after undergoing cancer treatment with radiotherapy, considering that most subjects, prior to treatment, received indication of dental extraction to avoid osteoradionecrosis [15]. Therefore, only the use of six month of radiotherapy is being 
Table 3: Comparison of molar bite force $(\mathrm{N})$ and standard error ( $t$-test) between Group I and Group II in the molar region.

\begin{tabular}{|c|c|c|c|}
\hline Bite force & Groups & Mean Standard Error & $\boldsymbol{p}$ \\
\hline \multirow{2}{*}{ Right Molar } & I & $490 \pm 60$ & \multirow{2}{*}{0.59} \\
\cline { 2 - 4 } & II & $420 \pm 64$ & \\
\hline \multirow{2}{*}{ Left Molar } & I & $481 \pm 60$ & \multirow{2}{*}{0.37} \\
\cline { 2 - 4 } & II & $451 \pm 68$ & \\
\hline
\end{tabular}

Significant $(p<0.05)$

evaluated as a factor of influence on the performance of the stomatognathic system.

Radiotherapy has deleterious effects on teething in children and adolescents, such as hypodontia, microdontia, altered eruption patterns, and tooth dwarfism. These changes usually occur in individuals under the age of six, where the root surface area decreases due to exposure to radiation, causing the early loss of tooth [16].

The study sample included individuals with ages ranging from 15 to 55 years (only one was 15 and another 16 years old), therefore, it was not possible to observe changes in dentition.

The non-standardized cancer location introduced a possible bias in the results obtained, because each individual evaluated had different locations and types of tumors in the head and neck region, with individualized treatment prescription that were established according to the needs of each individual, with the dosage of irradiation, the irradiated area and number of sessions specific to each type of injury. These data were statistically analyzed to define whether they would interfere on the results obtained. They were considered statistically insignificant, but anyway, they are factors of differentiation. The use of radiotherapy as a treatment was the common factor, following the standards set by the oncologists.

Individuals submitted to the treatment of head and neck cancer usually present depressive symptoms associated with the impairment of masticatory ability caused by the treatment, which can determine changes in the temporomandibular joint, loss and alteration of the masticatory system, loss of teeth i.e. general changes in the oral anatomy $[7,16,17]$.

In this study, it was possible to observe such depressive conditions. Although patients were fully dentate, they reported numerous complaints, most of them related to pain and loss of masticatory function. Reports of discomfort of individuals are based on the electromyography results obtained, which showed greater muscle activation in all clinical conditions evaluated, including at rest, being consistent with individuals with muscular disorders [17-19].

Maintaining proper oral health is as important as planning the treatment of oral cavity cancers, as they are essential to minimize different oral cavity diseases and to avoid more complex surgical interventions, especially when radiation therapy is one of the choice of treatment [7].

Preventive programs of oral hygiene should be initiated prior to radiotherapy. The clinical examination should be performed as soon as possible, since it is not convenient to postpone the oncological treatment.

The teeth with great destruction by caries, with pulpal infection, impacted partly or even teeth proximal to the tumor and deciduous teeth with physiological rizolisis, as well as teeth affected by periodontal disease, extensive periapical lesions and radicular remains should be removed [16].

Tooth loss reduces the number of dental contacts and can cause difficulty in mastication, formation of bolus in preparation for the swallow, and digestion of food [7]. In this study, this was considered a controlled factor for fully dentate individuals, except the third molars, which probably shows that the variations found in the functions of the stomatognathic system could somehow be related to radiation therapy, which is an important local treatment modality. The deleterious effects on tissues after the application of radiation are still unknown, for example, the myoelectric activity, bite force and masticatory muscles thickness.

During the electromyographic examinations, it was observed that the activation behavior of muscle fibers showed a disorganized pattern when compared to the control group. Patients with head and neck cancer evaluated in the present study showed increased electromyographic activity when compared to the control group. This finding is a strong indication of muscular hyperactivity, especially when the electrical activity is evaluated at rest. Several authors report that an increased electrical activity of the masseter and temporal muscles can be a sign of temporomandibular and/or muscular dysfunction $[19,20]$.

In addition to an increased electromyographic activity of the masticatory muscles and bite force of individuals treated for head and neck cancer when compared to controls, an increase in the muscle thickness measures of these individuals was also observed. There is evidence of an association between the activity of the masticatory muscles, the area and the thickness of these muscles [21-24].

The ultrasound examination of the masticatory muscles is a reproducible and easy-to-use method to obtain the function patterns of the masticatory muscles that can be directly correlated with the bite force $[25,26]$. The results obtained in this study are consistent with these findings. Despite an apparent impairment in the function of the masticatory muscles of individuals exposed to radiation treatments, there was an increase in bite force measures when compared to data collected from controls.

Increased muscle thickness in patients treated in this study may have occurred due to the unbalanced function of these muscles during chewing, which is inherent to the mastication of individuals undergoing radiation treatment. This imbalance is a disordered activation of the muscle fibers during tasks such as chewing, which recruits a great number of fibers to perform, when compared to individuals with normal function.

This overuse of muscle fibers during mastication may be responsible for a muscular hypertrophy determined by an increased muscle thickness [21-24]. 
Thus, further studies should be carried out to determine the most interfering factors related to treatments of head and neck tumors so that these treatments can be improved without having any influence on the patient's chances of survival, and therefore, provide better quality of life.

\section{Acknowledgments}

The authors are grateful to Fundacao de Amparo a Pesquisa do Estado de Sao Paulo (FAPESP, Brazil).

\section{References}

1. Filho VW. The epidemiology of laryngeal cancer in Brazil. Sao Paulo Med J. 2004; 122(5):188-94.

2. Laban S, Atanackovic D, Luetkens T, Knecht R, Busch CJ, Freytag M, et al. Simultaneous cytoplasmic and nuclear protein expression of MAGE-A family and NY-ESO-1 cancer-testis antigens represents an independent marker for poor survival in head \& neck cancer. Int J Cancer. 2014; 135(5):1142-52. doi: 10.1002/ijc.28752.

3. Hashibe M, Sturgis EM. Epidemiology of oral-cavity and oropharyngeal carcinomas: controlling a tobacco epidemic while a human papillomavirus epidemic emerges. Otolaryngol Clin North Am. 2013; 46(4):507-20. doi: 10.1016/j.otc.2013.05.001.

4. Alcalay RN, Shulman JM, Plotkin SR. Ramsay Hunt syndrome in a patient with metastatic lung cancer to brain. J Neurooncol. 2008; 86(1):55-56.

5. Goiato MC, Haddad MF, dos Santos DM, Pesqueira AA, Filho HG, Pellizzer EP. Incidents malignant neoplasias maxillofacial area. J Craniofac Surg. 2009; 20(4):1210-3. doi: 10.1097/SCS.0b013e3181acdd89.

6. Farnebo L, Tiefenbock K, Ansell A, Thunell LK, Garvin S, Roberg K. Strong expression of survivin is associated with positive response to radiotherapy and improved overall survival in head and neck squamous cell carcinoma patients. Int J Cancer. 2013; 133(8):1994-2003. doi: 10.1002/ijc.28200.

7. Pace-Balzan A, Shaw RJ, Butterworth C. Oral rehabilitation following treatment for oral cancer. Periodontol 2000. 2011; 57(1):102-17. doi: 10.1111/j.1600-0757.2011.00384.x.

8. Hermens HJ, Freriks B, Merletti R. European recommendations for surface electromyography, Roessingh Research and Development B.V., Results of SENIAN project. Enschede, the Netherlands. 1999.

9. Regalo SC, Santos CM, Vitti M, Regalo CA, de Vasconcelos PB, Mestriner $\mathrm{W}$ Jr, et al. Evaluation of molar and incisor bite force in indigenous compared with white population in Brazil. Arch Oral Biol. 2008; 53(3):2826.

10. Palinkas M, Nassar MS, Cecílio FA, Siéssere S, Semprini M, Machadode-Sousa JP, et al. Age and gender influence on maximal bite force and masticatory muscles thickness. Arch Oral Biol. 2010; 55(10):797-802. doi: 10.1016/j.archoralbio.2010.06.016.

11. Kogawa EM, Calderon PS, Lauris JR, Araujo CR, Conti PC. Evaluation of maximal bite force in temporomandibular disorders patients. J Oral Rehabil. 2006; 33(8):559-65.
12. De Rossi M, Santos CM, Migliorança R, Regalo SC. All on Four ${ }^{\circledR}$ Fixed Implant Support Rehabilitation: A Masticatory Function Study. Clin Implant Dent Relat Res. 2014; 16(4):594-600. doi: 10.1111/cid.12031.

13. Borges Tde F, Regalo SC, Taba M Jr, Siéssere S, Mestriner W Jr, Semprini $\mathrm{M}$. Changes in masticatory performance and quality of life in individuals with chronic periodontitis. J Periodontol. 2013; 84(3):325-31. doi: 10.1902/ jop.2012.120069.

14.INCA. 2013; Available from: http://www1.inca.gov.br/english.

15. Khojastepour L, Bronoosh P, Zeinalzade M. Mandibular bone changes induced by head and neck radiotherapy. Indian J Dent Res. 2012; 23(6):774-7. doi: 10.4103/0970-9290.111258.

16. Goiato MC, dos Santos DM, Haddad MF, Pesqueira AA, de Carvalho Dekon SF, Zavanelli AC. Most Frequent Tumors in Maxillofacial Area Rehabilitated Through Surgical Reconstruction and Prostheses. JCraniofac Surg. 2010; 21(2):396-9. doi: 10.1097/SCS.0b013e3181cfa70c.

17. Koga DH, Salvajoli JV, Alves FA. Dental extractions and radiotherapy in head and neck oncology: review of the literature. Oral Dis. 2008; 14(1):404. doi: 10.1111/j.1601-0825.2006.01351.x.

18. Ferrario VF, Sforza C, Tartaglia GM, Dellavia C. Immediate effect of a stabilization splint on masticatory muscle activity in temporomandibular disorder patients. J Oral Rehabil. 2002; 29(9): 810-5.

19. Rancan SV, Bataglion C, Bataglion SA, Bechara OM, Semprini M, Siéssere $S$, et al. Acupuncture and temporomandibular disorders: a 3-month follow-up EMG study. J Altern Complement Med. 2009; 15(12):1307-10. doi: $10.1089 / \mathrm{acm} .2009 .0015$.

20. Siéssere S, Sousa LG, Lima Nde A, Semprini M, de Felício CM, Watanabe PC, et al. Electromyographic activity of masticatory muscles in women with osteoporosis. Braz Dent J. 2009; 20(3): 237-42.

21. Bakke M, Tuxen A, Vilmann P, Jensen BR, Vilmann A,Toft M, et al. Ultrasound image of human masseter muscle related to bite force, electromyography, facial morphology, and occlusal factors. Scand J Dent Res. 1992; 100(3):164-71.

22. Ruf S, Pancherz H, Kirschbaum M. Facial morphology and the size and activity of the masseter muscle. Fortschr Kieferorthop. 1994; 55(5):219-27.

23. Bertram S, Brandlmaier I, Rudisch A, Bodner G, Emshoff R. Crosssectional characteristics of the masseter muscle: an ultrasonographic study. Int J Oral Maxillofac Surg. 2003; 32(1):64-8.

24. Georgiakaki I, Tortopidis D, Garefis P, Kiliaridis S. Ultrasonographic thickness and electromyographic activity of masseter muscle of human females. J Oral Rehabil. 2007; 34(2):121-8.

25. Raadsheer MC, Kiliaridis S, Van Eijden TM, Van Ginkel FC, PrahlAndersen B. Masseter muscle thickness in growing individuals and its relation to facial morphology. Arch Oral Biol. 1996; 41(4):323-32.

26. Karakis D, Aktas-Yilmaz B, Dogan A, Yetkin I, Bek B. The bite force and craniofacial morphology in patients with acromegaly: A pilot study. Med Oral Patol Oral Cir Bucal. 2014; 19(1):e1-7. 